\title{
A Maillet Type Theorem for First Order Singular Nonlinear Partial Differential Equations
}

\author{
By
}

Akira SHIRAI*

\begin{abstract}
We shall study the first order singular nonlinear partial differential equation of the form $f\left(x, u(x), \partial_{x} u(x)\right)=0$ with $u(0)=0$, where $x \in \mathbf{C}^{n}$ and $f(x, u, \xi)$ is holomorphic in a neighborhood of the origin. This equation is said to be singular if $f(0,0, \xi) \equiv 0$ for all $\xi \in \mathbf{C}^{n}$. The purpose of this paper is to study the Maillet type theorem which means to determine the Gevrey order of a formal power series solution which may diverge.
\end{abstract}

\section{$\S 1$. Introduction}

We consider the following first order nonlinear partial differential equation of general form in the complex domain:

$$
\left\{\begin{array}{l}
f\left(x, u(x), \partial_{x} u(x)\right)=0, \\
u(0)=0
\end{array}\right.
$$

where $x=\left(x_{1}, \ldots, x_{n}\right) \in \mathbf{C}^{n}, \partial_{x} u=\left(\partial_{x_{1}} u, \ldots, \partial_{x_{n}} u\right)$, and $f(x, u, \xi)(\xi=$ $\left.\left(\xi_{1}, \ldots, \xi_{n}\right) \in \mathbf{C}^{n}\right)$ is a holomorphic function in a neighborhood of the origin.

We assume that $f(x, u, \xi)$ is an entire function in $\xi$ variables when $x$ and $u$ are fixed. As a fundamental assumption, we always assume the existence of a formal solution of the equation (1.1), that is,

Communicated by T. Kawai. Received January 7, 2002. Revised April 2, 2002.

2000 Mathematics Subject Classification(s): Primary 35F20; Secondary 35A20, 35C10.

*Graduate School of Mathematics, Nagoya University, Nagoya 464-8602, Japan.

e-mail: m96034q@math.nagoya-u.ac.jp 
Assumption 1. The equation (1.1) has a formal solution of the form

$$
u(x)=\sum_{|\alpha| \geq 1} u_{\alpha} x^{\alpha}=\sum_{j=1}^{n} \xi_{j}^{0} x_{j}+\sum_{|\alpha| \geq 2} u_{\alpha} x^{\alpha} \in \mathbf{C}[[x]],
$$

where $\alpha=\left(\alpha_{1}, \ldots, \alpha_{n}\right) \in \mathbf{N}^{n}(\mathbf{N}=\{0,1,2, \ldots\})$ denotes the multi-index and $|\alpha|=\alpha_{1}+\cdots+\alpha_{n}$.

Our interest in this paper is to study the convergence or the divergence nature of such a formal solution in the case where the equation (1.1) is singular in the sense defined in Miyake-Shirai [3] as follows:

$$
f(0,0, \xi) \equiv 0, \quad \text { for all } \xi \in \mathbf{C}^{n} .
$$

By $(1.3)$, the coefficients $\xi^{0}=\left(\xi_{1}^{0}, \ldots, \xi_{n}^{0}\right)$ of linear part of the formal solution (1.2) satisfy

$$
\left.\frac{\partial}{\partial x_{i}} f\left(x, u(x), \partial_{x} u(x)\right)\right|_{x=0}=\frac{\partial f}{\partial x_{i}}\left(0,0, \xi^{0}\right)+\frac{\partial f}{\partial u}\left(0,0, \xi^{0}\right) \xi_{i}^{0}=0
$$

for $i=1,2, \ldots, n$. We take and fix one $\xi^{0}$ of such roots.

Let $v(x)=u(x)-\sum_{j=1}^{n} \xi_{j}^{0} x_{j}$ be a new unknown function. By substituting this power series into (1.1), we see that $v(x)$ satisfies the following equation:

$$
\left\{\begin{array}{l}
P_{0} v(x)=\sum_{|\alpha|=2} c_{\alpha} x^{\alpha}+f_{3}\left(x, v(x), \partial_{x} v(x)\right), \\
v(x)=O\left(|x|^{2}\right)
\end{array}\right.
$$

where $f_{3}(x, v, \xi)$ is holomorphic in a neighborhood of the origin with Taylor expansion

$$
f_{3}(x, v, \xi)=\sum_{|\alpha|+2 r+|\kappa| \geq 3} f_{\alpha r \kappa} x^{\alpha} v^{r} \xi^{\kappa}, \quad \kappa=\left\{\kappa_{j}\right\} \in \mathbf{N}^{n}, \quad|\kappa|=\sum_{j=1}^{n} \kappa_{j},
$$

and $P_{0}$ denotes the operator of the form

$$
P_{0}=\left(x_{1}, \ldots, x_{n}\right) A\left(\begin{array}{c}
\partial_{x_{1}} \\
\vdots \\
\partial_{x_{n}}
\end{array}\right)+f_{u}\left(0,0, \xi^{0}\right)
$$

with an $n \times n$ matrix $A=\left(a_{i j}\right)_{i, j=1,2, \ldots, n}$ given by

$$
a_{i j}=\frac{\partial^{2} f}{\partial x_{i} \partial \xi_{j}}\left(0,0, \xi^{0}\right)+\frac{\partial^{2} f}{\partial u \partial \xi_{j}}\left(0,0, \xi^{0}\right) \xi_{i}^{0}
$$


Let the Jordan canonical form of $A$ be given by

$$
A \sim\left(\begin{array}{ccccc}
A_{m} & & & & \\
& B_{1} & & & \\
& & \ddots & & \\
& & & B_{p} & \\
& & & & O_{q}
\end{array}\right)
$$

where

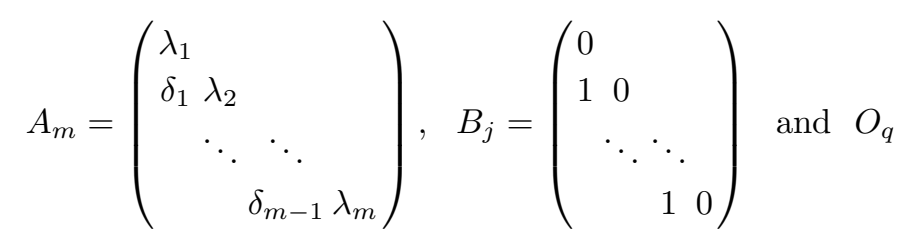

are the block of nonzero eigenvalues of size $m$, nilpotent block of size $n_{j}$ and zero matrix block of size $q$, respectively. It is obvious that $m+n_{1}+\cdots+n_{p}+q=n$.

Under the above situation, Miyake-Shirai [3] proved the following results:

Theorem 1 (Miyake-Shirai). (i) Let $m=n$ and $\left\{\lambda_{j}\right\}_{j=1}^{n}$ satisfy the following condition which is called the Poincaré condition:

$$
\operatorname{Ch}\left(\lambda_{1}, \ldots, \lambda_{n}\right) \not \supset 0,
$$

where $\operatorname{Ch}\left(\lambda_{1}, \ldots, \lambda_{n}\right)$ denotes the convex hull of $\left\{\lambda_{1}, \ldots, \lambda_{n}\right\}$. Then the formal solution $u(x)$ converges in a neighborhood of the origin.

(ii) If $q=n$ and $f_{u}\left(0,0, \xi^{0}\right) \neq 0$, then the formal solution $u(x)$ belongs to the Gevrey class of order at most 2 , that is, the power series $\sum_{|\alpha| \geq 1} u_{\alpha} x^{\alpha} /|\alpha|$ ! converges in a neighborhood of the origin.

In this paper, we give a refinement of Theorem 1. Firstly we determine the Gevrey order of the formal solution $u(x)$ in the case where the matrix $A$ is nilpotent, that is, the case where $m=0$ and $p \geq 1$, which is not studied in Miyake-Shirai [3].

Theorem 2. If $m=0, p \geq 1$ and $f_{u}\left(0,0, \xi^{0}\right) \neq 0$, then the formal solution $u(x)$ of (1.1) belongs to the Gevrey class of order at most $2 N$ with $N=$ $\max \left\{n_{1}, \ldots, n_{p}\right\}$, that is, the power series $\sum_{|\alpha| \geq 1} u_{\alpha} x^{\alpha} /|\alpha| !^{2 N-1}$ converges in a neighborhood of the origin.

In the case of first order linear singular equations, Hibino [2] and Yamazawa [5], [6] studied the same problem and they determined the Gevrey order of the 
formal solutions which deeply depends on the Jordan canonical form of $A$. Theorem 2 is a nonlinear version of their results in the case where the matrix $A$ is nilpotent.

Secondly we consider (1.1) in the case where $A$ is regular, that is, the case where $m=n$. By Theorem 1, we already know that the formal solution converges in a neighborhood of the origin under the Poincaré condition (1.6). In this paper, we assume the following condition which is a combination of the Siegel condition and the non-resonance condition:

Assumption 2. For all $\alpha=\left\{\alpha_{j}\right\} \in \mathbf{N}^{n}$ with $|\alpha| \geq 2$, there exist $C>0$ and $p<1$ such that

$$
\left|\sum_{j=1}^{n} \lambda_{j} \alpha_{j}+f_{u}\left(0,0, \xi^{0}\right)\right| \geq C|\alpha|^{p}
$$

We remark that in the case where $p>0$, the condition is equivalent to the following two conditions:

(a) $\left|\sum_{j=1}^{n} \lambda_{j} \alpha_{j}\right| \geq C|\alpha|^{p}, \quad|\alpha| \geq 2, \quad$ (Siegel condition),

(b) $\sum_{j=1}^{n} \lambda_{j} \alpha_{j}+f_{u}\left(0,0, \xi^{0}\right) \neq 0, \quad|\alpha| \geq 2, \quad$ (Non-resonance condition).

The Jordan canonical form of $A$ in (1.5) is written by

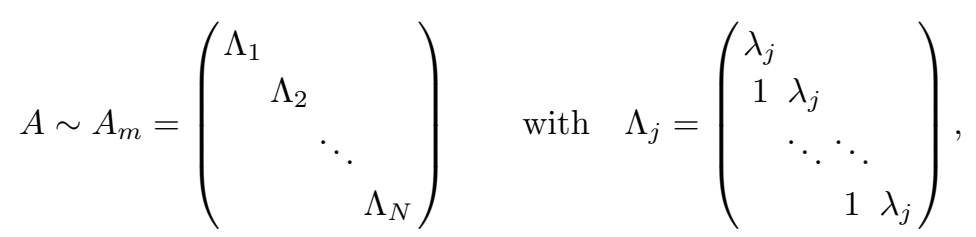

where $\lambda_{j} \neq 0$ for $j=1,2, \ldots, N$ and $k_{j} \in \mathbf{N}$ denotes the size of $\Lambda_{j}$.

Our result is stated as follows:

Theorem 3. Under Assumption 2, the formal solution of the equation (1.1) belongs to the Gevrey class of order at most $s$ with

$$
s=2 \max \left\{k_{1}, k_{2}, \ldots, k_{N}\right\}(1-p)+p,
$$

that is, the power series $\sum_{|\alpha| \geq 1} u_{\alpha} x^{\alpha} /|\alpha| !^{s-1}$ converges in a neighborhood of the origin.

We remark that when $p=1$ Theorem 3 claims the convergence of the formal solution, which is already known by Theorem 1 . 
At the end of this introduction we give a mention about the study by Gérard-Tahara on singular partial differential equations which can be seen in their book [1] and the references therein. Their research treats many kinds of problems for singular (nonlinear) partial differential equations such as the convergence of formal solutions, the Maillet type theorem for divergent formal solutions, the existence of singular solutions, etc. However, their study is somewhat restricted to the equations of reduced form such as

$$
\left\{\begin{array}{l}
\sum_{i, j=1}^{n} a_{i j} x_{i} \partial_{x_{j}} u+c u=\sum_{j=1}^{n} a_{j} x_{j}+f_{2}\left(x, u,\left\{x_{i} \partial_{x_{j}} u\right\}_{i, j=1,2, \ldots, n}\right), \\
u(0)=0
\end{array}\right.
$$

where $f_{2}(x, u, \xi)=\sum_{|\alpha|+r+|\kappa| \geq 2} f_{\alpha r \kappa} x^{\alpha} u^{r} \xi^{\kappa}$. Our equation (1.4) deals with a wider class of equations than (1.9) in the sense that the derivative of the unknown function $u$ appears in nonlinear terms in the form $\partial_{x} v$, not in the form $\left\{x_{i} \partial_{x_{j}} u\right\}_{i, j=1,2, \ldots, n}$ as in (1.9). For example,

$$
\sum_{i, j=1}^{2} a_{i j} x_{i} \partial_{x_{j}} u+u=\left(x_{1}+x_{2}\right)^{2}+u \partial_{x_{2}} u, \quad u=O\left(|x|^{2}\right)
$$

can be treated only by our framework. Our theory can be said to be a trial toward the classification of singular equations from the general point of view.

\section{$\S 2 . \quad$ Refinements of Theorems}

The estimate of Gevrey order given in Theorems 2 and 3 is not bestpossible. In fact, if we introduce the Gevrey order $s=\left(s_{1}, \ldots, s_{n}\right)$ depending on the variables (cf. Definition 1 below) after employing a linear transformation which reduces the matrix $A$ to its Jordan canonical form, we can obtain more precise estimates.

Definition 1 ( $\boldsymbol{s}$-Borel transformation). Let $\boldsymbol{s}=\left(s_{1}, \ldots, s_{n}\right) \in\left(\mathbf{R}_{\geq 1}\right)^{n}$ where $\mathbf{R}_{\geq 1}=\{x \in \mathbf{R} ; x \geq 1\}$. For a formal power series $f(x)=\sum_{|\alpha| \geq 0} f_{\alpha} x^{\alpha}$, the $\boldsymbol{s}$-Borel transform $\mathcal{B}_{\boldsymbol{s}}(f)(x)$ of $f(x)$ is defined by

$$
\mathcal{B}_{\boldsymbol{s}}(f)(x)=\sum_{|\alpha| \geq 0} f_{\alpha} \frac{|\alpha| !}{(\boldsymbol{s} \cdot \alpha) !} x^{\alpha}
$$


Definition 2 (Gevrey class $\mathcal{G}_{x}^{\boldsymbol{s}}$ ). We say that $f(x)=\sum_{|\alpha| \geq 0} f_{\alpha} x^{\alpha} \in$ $\mathcal{G}_{x}^{\boldsymbol{s}}$, if the $\boldsymbol{s}$-Borel transform $\mathcal{B}_{\boldsymbol{s}}(f)(x)$ converges in a neighborhood of the origin, and $s$ is called the Gevrey order.

Remark 1. (i) If two Gevrey orders $s=\left\{s_{j}\right\}$ and $\boldsymbol{s}^{\prime}=\left\{s_{j}^{\prime}\right\}$ satisfy $s_{j} \leq s_{j}^{\prime}$ for all $j=1,2, \ldots, n$, then $\mathcal{G}_{x}^{\boldsymbol{s}} \subset \mathcal{G}_{x}^{\boldsymbol{s}^{\prime}}$.

(ii) If $\boldsymbol{s}^{\prime}=\left(s^{\prime}, s^{\prime}, \ldots, s^{\prime}\right) \in\left(\mathbf{R}_{\geq 1}\right)^{n}$, then $f(x) \in \mathcal{G}_{x}^{\boldsymbol{s}^{\prime}}$ if and only if

$$
\sum \frac{f_{\alpha}}{|\alpha| !^{\prime}-1} x^{\alpha}
$$

converges in a neighborhood of the origin. Moreover, for all linear transformations $\xi=x M\left(\xi \in \mathbf{C}^{n}\right.$ and $M$ is an $n \times n$ invertible matrix), $g(\xi):=f\left(\xi M^{-1}\right) \in$ $\mathcal{G}_{\xi}^{\left(\boldsymbol{s}^{\prime}\right)}$.

(iii) For a formal power series $u(x) \in \mathbf{C}[[x]]$, if $\mathcal{B}_{\boldsymbol{s}}(u)(x) \in \mathcal{G}_{x}^{\widetilde{\boldsymbol{s}}}$, then we have $u(x) \in \mathcal{G}_{x}^{\boldsymbol{s}+\widetilde{\boldsymbol{s}}-\mathbf{1}_{n}}$ with $\mathbf{1}_{n}=(1,1, \ldots, 1) \in \mathbf{N}^{n}$.

Let us give a refined form of Theorem 2. Let us suppose that the same assumption as in Theorem 2 holds and further that the vanishing order of $v(x)$ is $K \geq 2$. Then by a linear change of independent variables which brings the matrix $A$ in (1.5) to the Jordan canonical form, the equation (1.4) is reduced to the following form:

$$
\left\{\begin{array}{l}
P v(y, z)=\sum_{|\beta|+|\gamma|=K} c_{\beta \gamma} y^{\beta} z^{\gamma}+f_{K+1}\left(y, z, v, \partial_{y} v, \partial_{z} v\right) \\
v(y, z)=O\left((|y|+|z|)^{K}\right)
\end{array}\right.
$$

where

$$
P=\sum_{i=1}^{p} \sum_{j=1}^{n_{i}-1} \delta y_{i, j+1} \partial_{y_{i, j}}+c, \quad c=f_{u}\left(0,0, \xi^{0}\right)
$$

$\delta, c \in \mathbf{C} \backslash\{0\}, y=\left(\boldsymbol{y}^{1}, \boldsymbol{y}^{2}, \ldots, \boldsymbol{y}^{p}\right) \in \mathbf{C}^{n_{1}+\cdots+n_{p}}$ where $\boldsymbol{y}^{i}=\left(y_{i, 1}, \ldots, y_{i, n_{i}}\right) \in$ $\mathbf{C}^{n_{i}}, z=\left(z_{1}, \ldots, z_{q}\right) \in \mathbf{C}^{q}$,

$$
f_{K+1}(y, z, v, \eta, \zeta)=\sum_{|\beta|+|\gamma|+K r+(K-1)(|\mu|+|\nu|) \geq K+1} f_{\beta \gamma r \mu \nu} y^{\beta} z^{\gamma} v^{r} \eta^{\mu} \zeta^{\nu},
$$

where $|\beta|,|\gamma|,|\mu|$ and $|\nu|$ denote the length of multi-indices $\beta=\left\{\beta_{i, j}\right\} \in$ $\mathbf{N}^{n_{1}+\cdots+n_{p}}, \gamma=\left\{\gamma_{k}\right\} \in \mathbf{N}^{q}, \mu=\left\{\mu_{i, j}\right\} \in \mathbf{N}^{n_{1}+\cdots+n_{p}}$ and $\nu=\left\{\nu_{k}\right\} \in \mathbf{N}^{q}$, respectively. 
Remark 2. We may assume that the constant $\delta$ is as small as we want. Indeed, we introduce new independent variables $\widetilde{y}=\left\{\widetilde{y}_{i, j}\right\}$ by $\widetilde{y}_{i, j}=$ $\varepsilon^{n_{1}+\cdots+n_{i-1}+j} y_{i, j}$. Then $\delta$ is replaced by $\varepsilon \delta$. Therefore, by choosing $\varepsilon>0$ small enough, we may assume that the coefficient $\delta$ is arbitrary small.

For $\boldsymbol{p}=\left(p_{1}, p_{2}, \ldots, p_{d}\right)(d \geq 1)$ and a constant $a$, we define $\boldsymbol{p}(a)$ by

$$
\boldsymbol{p}(a)=\left(p_{1}+a, p_{2}+a, \ldots, p_{d}+a\right) .
$$

Then Theorem 2 is obtained immediately from the following:

Proposition 1. The equation (2.2) has a unique formal solution which belongs to the Gevrey class of order $s$ with

$$
\boldsymbol{s}=\left(\boldsymbol{s}^{1}(\sigma), \boldsymbol{s}^{2}(\sigma), \ldots, \boldsymbol{s}^{p}(\sigma), \mathbf{1}_{q}(\sigma)\right),
$$

where $\boldsymbol{s}^{i}=\left(1,2, \ldots, n_{i}\right) \in \mathbf{N}^{n_{i}}, \mathbf{1}_{q}=(1,1, \ldots, 1) \in \mathbf{N}^{q}$ and

$$
\begin{gathered}
\sigma=\max _{(\beta, \gamma, r, \mu, \nu)}\left\{\frac{A(\mu, \nu)}{|\beta|+|\gamma|+K r+(K-1)(|\mu|+|\nu|)-K} ; f_{\beta \gamma r \mu \nu} \neq 0\right\}, \\
A(\mu, \nu)=\left\{\begin{array}{cl}
\max \left\{j ; \mu_{i, j} \neq 0\right\} & \text { if }|\mu| \geq 1, \\
1 & \text { if }|\mu|=0,|\nu| \geq 1, \\
0 & \text { if }|\mu|=|\nu|=0
\end{array}\right.
\end{gathered}
$$

Proof of Theorem 2. As mentioned above, the equation (2.2) is the one which is obtained from (1.4) by a linear change of independent variables. The Gevrey order of the formal solution $v(x)$ of (1.4) is estimated by the maximal value of components of $s$. Because of the trivial estimate $A(\mu, \nu) \leq N=$ $\max \left\{n_{1}, \ldots, n_{p}\right\}$ and the determination of $\boldsymbol{s}^{i}$, we see that the Gevrey order of $v$ is estimated by $2 N$.

Next we shall suppose Assumption 2 in Section 1 and consider a refinement of Theorem 3. Similarly to the reduction of (1.4) into (2.2), the equation (1.4) is reduced to the following one by a linear change of independent variables.

$$
\left\{\begin{array}{l}
(\Lambda+\Delta) v(y)=\sum_{|\alpha|=K} c_{\alpha} y^{\alpha}+f_{K+1}\left(y, v(y), \partial_{y} v(y)\right) \\
v(x)=O\left(|y|^{K}\right), \quad K \geq 2
\end{array}\right.
$$

where

$$
\Lambda=\sum_{i=1}^{N} \sum_{j=1}^{k_{i}} \lambda_{i} y_{i, j} \partial_{y_{i, j}}+f_{u}\left(0,0, \xi^{0}\right), \quad \Delta=\sum_{i=1}^{N} \sum_{j=1}^{k_{i}-1} \delta y_{i, j+1} \partial_{y_{i, j}}
$$




$$
f_{K+1}(y, v, \xi)=\sum_{|\alpha|+K r+(K-1)|\kappa| \geq K+1} f_{\alpha r \kappa} y^{\alpha} v^{r} \xi^{\kappa} .
$$

By the same reason as in Remark 2, we may assume the coefficient $\delta$ is arbitrary small as we want.

Now Theorem 3 is obtained from the following:

Proposition 2. Under the condition (1.7), the equation (2.7) has a unique formal solution which belongs to the Gevrey class of order $\boldsymbol{s}$ with

$$
\boldsymbol{s}=\left(\boldsymbol{t}^{1}(\sigma), \ldots, \boldsymbol{t}^{N}(\sigma)\right)
$$

where $\boldsymbol{t}^{i}=\left\{s_{i, j}\right\}_{j=1, \ldots, k_{i}}=\{j-p(j-1)\}_{j=1, \ldots, k_{i}}$ and

$$
\begin{array}{r}
\sigma=\max _{(\alpha, r, \kappa)}\left\{\frac{A(r, \kappa)}{|\alpha|+K r+(K-1)|\kappa|-K} ; f_{\alpha r \kappa} \neq 0\right\}, \\
A(r, \kappa)=\left\{\begin{array}{cl}
\max _{i, j}\left\{s_{i, j}-p ; \kappa_{i, j} \neq 0\right\} & \text { if }|\kappa| \geq 1, \\
\max \{-p, 0\} & \text { if } r \geq 1,|\kappa|=0, \\
0 & \text { if } r=|\kappa|=0 .
\end{array}\right.
\end{array}
$$

Proof of Theorem 3. By an easy calculation, we have

$$
\sigma \leq \max \left\{s_{i, j}\right\}-p \leq \max \left\{k_{1}, \ldots, k_{N}\right\}(1-p) .
$$

Then $s_{i, j}+\sigma$ are estimated by

$$
\begin{aligned}
s_{i, j}+\sigma & \leq \max \left\{s_{i, j}\right\}+\max \left\{k_{1}, \ldots, k_{N}\right\}(1-p) \\
& \leq 2 \max \left\{k_{1}, \ldots, k_{N}\right\}(1-p)+p
\end{aligned}
$$

By this inequality, we can see that the formal solution belongs to the Gevrey class of order at most

$$
s=2 \max \left\{k_{1}, \ldots, k_{N}\right\}(1-p)+p .
$$

\section{$\S 3 . \quad$ Proof of Propositions 1 and 2}

In this section, we shall prove Propositions 1 and 2 by assuming the lemmas below which will be proved in the next section. 


\section{§3.1. Proof of Proposition 1}

First we prove the uniqueness of formal solutions. We decompose the formal power series $v(y, z)$ into the sum of homogeneous polynomials in $(y, z)$ variables, that is, we put $v(y, z)=\sum_{L \geq K} v_{L}(y, z)$ where $v_{L}(y, z)$ is a homogeneous polynomial of degree $L$ in $(y, z)$ variables.

By substituting $v(y, z)=\sum_{L \geq K} v_{L}(y, z)$ into the equation, we can see that the homogeneous polynomials $\left\{v_{L}(y, z)\right\}_{L \geq K}$ satisfy the following relations:

$$
P v_{K}(y, z)=\sum_{|\beta|+|\gamma|=K} c_{\beta \gamma} y^{\beta} z^{\gamma},
$$

and for $L>K$

$$
\begin{aligned}
P v_{L}(y, z)= & \sum^{\prime} f_{\beta \gamma r \mu \nu} y^{\beta} z^{\gamma} \prod_{l=1}^{r} v_{L_{l}}(y, z) \\
& \times \prod_{i=1}^{p} \prod_{j=1}^{n_{i}} \prod_{l=1}^{\mu_{i, j}} \partial_{y_{i, j}} v_{M_{i j l}}(y, z) \prod_{k=1}^{q} \prod_{l=1}^{\nu_{k}} \partial_{z_{k}} v_{N_{k l}}(y, z),
\end{aligned}
$$

where $P=\sum_{i=1}^{p} \sum_{j=1}^{n_{i}-1} \delta y_{i, j+1} \partial_{y_{i, j}}+c$ and $\sum^{\prime}$ is taken over

$$
\left\{\begin{array}{l}
|\beta|+|\gamma|+K r+(K-1)(|\mu|+|\nu|) \geq K+1 \\
|\beta|+|\gamma|+\sum_{l=1}^{r} L_{l}+\sum_{i=1}^{p} \sum_{j=1}^{n_{i}} \sum_{l=1}^{\mu_{i, j}}\left(M_{i j l}-1\right)+\sum_{k=1}^{q} \sum_{l=1}^{\nu_{k}}\left(N_{k l}-1\right)=L
\end{array}\right.
$$

It follows from (3.3) that $L_{l}, M_{i j l}, N_{k l} \leq L-1$ for all $i, j, k$ and $l$. Indeed, for $M_{i j l}$ we have

$$
\begin{aligned}
L & =|\beta|+|\gamma|+\sum_{l=1}^{r} L_{l}+\sum_{i=1}^{p} \sum_{j=1}^{n_{i}} \sum_{l=1}^{\mu_{i, j}}\left(M_{i j l}-1\right)+\sum_{k=1}^{q} \sum_{l=1}^{\nu_{k}}\left(N_{k l}-1\right) \\
& \geq|\beta|+|\gamma|+K r+(K-1)(|\mu|+|\nu|)+\left(M_{i j l}-1\right)-(K-1) \\
& \geq M_{i j l}+1
\end{aligned}
$$

which implies $M_{i j l} \leq L-1$. We can prove the statements for $\left\{L_{l}\right\}$ and $\left\{N_{k l}\right\}$ in a similar mannar. These guarantee that (3.2) gives a recursion formula for $\left\{v_{L}\right\}$.

Here we prepare the following lemma:

Lemma 1. (i) Let $\mathbf{C}[y, z]_{L}$ be the set of homogeneous polynomials of degree $L$ in $y$ and $z$ variables. Then for all $L \geq 2$, the operator $P: \mathbf{C}[y, z]_{L} \longrightarrow$ $\mathbf{C}[y, z]_{L}$ is invertible. 
(ii) Let $\widehat{\boldsymbol{s}}:=\left(\boldsymbol{s}^{1}, \ldots, \boldsymbol{s}^{p}, \mathbf{1}_{q}\right)=\boldsymbol{s}-\boldsymbol{\sigma}_{n}$ with $\boldsymbol{\sigma}_{n}=\mathbf{1}_{n}(\sigma)-\mathbf{1}_{n}=(\sigma, \sigma, \ldots, \sigma)$ $\in\left(\mathbf{R}_{\geq 1}\right)^{n}$ where $\boldsymbol{s}^{i}$ is the vector defined in Proposition 1 , and let $u_{L}(y, z)$, $f_{L}(y, z) \in \mathbf{C}[y, z]_{L}$. We consider the following equation:

$$
P u_{L}(y, z)=f_{L}(y, z) .
$$

If a majorant relation $\mathcal{B}_{\widehat{\boldsymbol{s}}}\left(f_{L}\right)(y, z) \ll F_{L} \times(|y|+|z|)^{L}$ does hold with $|y|=$ $\sum_{i=1}^{p} \sum_{j=1}^{n_{i}} y_{i, j}$ and $|z|=\sum_{k=1}^{q} z_{k}$ by a positive constant $F_{L}>0$, then there exists a positive constant $C>0$ independent of $L$ such that

$$
\mathcal{B}_{\widehat{\boldsymbol{s}}}\left(u_{L}\right)(y, z)=\mathcal{B}_{\widehat{\boldsymbol{s}}}\left(P^{-1} f_{L}\right)(y, z) \ll C F_{L} \times(|y|+|z|)^{L} .
$$

By Lemma 1 (i), the recursion formulas (3.1) and (3.2) are solvable uniquely. Therefore the uniqueness follows immediately.

Next we shall give the estimate of the Gevrey order. We take $U(y, z)=$ $P v(y, z)$ as a new unknown function. Then $U(y, z)$ satisfies the following equation:

$$
\left\{\begin{array}{l}
U(y, z)=\sum_{|\beta|+|\gamma|=K} c_{\beta \gamma} y^{\beta} z^{\gamma}+f_{K+1}\left(y, z, P^{-1} U, \partial_{y} P^{-1} U, \partial_{z} P^{-1} U\right) \\
U(y, z)=O\left((|y|+|z|)^{K}\right)
\end{array}\right.
$$

By applying the $\widehat{\boldsymbol{s}}$-Borel transformation to the equation (3.5), we have

$$
\begin{aligned}
\mathcal{B}_{\widehat{\boldsymbol{s}}}(U)(y, z)= & \sum_{|\beta|+|\gamma|=K} c_{\beta \gamma} \frac{(|\beta|+|\gamma|) !}{\{\widehat{\boldsymbol{s}} \cdot(\beta, \gamma)\} !} y^{\beta} z^{\gamma} \\
& +\mathcal{B}_{\widehat{\boldsymbol{s}}}\left\{f_{K+1}\left(y, z, P^{-1} U, \partial_{y} P^{-1} U, \partial_{z} P^{-1} U\right)\right\}
\end{aligned}
$$

In order to construct a majorant equation of this equation, we prepare the following lemma.

Lemma 2. (i) For two arbitrary formal power series $u(y, z)=$ $\sum_{|\beta|+|\gamma| \geq 0} u_{\beta \gamma} y^{\beta} z^{\gamma}$ and $v(y, z)=\sum_{|\beta|+|\gamma| \geq 0} v_{\beta \gamma} y^{\beta} z^{\gamma}$, we have

$$
\mathcal{B}_{\widehat{\boldsymbol{s}}}(u v)(y, z) \ll C_{0} \mathcal{B}_{\widehat{\boldsymbol{s}}}(|u|)(y, z) \mathcal{B}_{\widehat{\boldsymbol{s}}}(|v|)(y, z), \quad C_{0}=\max \left\{s_{i j}\right\} \geq 1,
$$

where $|u|(y, z):=\sum_{|\beta|+|\gamma| \geq 0}\left|u_{\beta \gamma}\right| y^{\beta} z^{\gamma}$.

(ii) If $\mathcal{B}_{\widehat{\boldsymbol{s}}}(u)(y, z) \ll W(T)=\sum_{L \geq 0} W_{L} T^{L}(T=|y|+|z|)$, then there exists a positive constant $M>0$ independent of $i, j$ and $k$ such that

$$
\begin{array}{ll}
\mathcal{B}_{\widehat{\boldsymbol{s}}}\left(\partial_{y_{i, j}} P^{-1} u\right)(y, z) \ll M \frac{d}{d T}\left(T \frac{d}{d T}\right)^{j-1} W(T), & \text { for } 1 \leq i \leq p, 1 \leq j \leq n_{i}, \\
\mathcal{B}_{\widehat{\boldsymbol{s}}}\left(\partial_{z_{k}} P^{-1} u\right)(y, z) \ll M \frac{d}{d T} W(T), & \text { for } k=1,2, \ldots, q .
\end{array}
$$


Here we consider the following ordinary differential equation which is called the majorant equation of (3.6):

$$
\begin{aligned}
& W(T)=\left(\sum_{|\beta|+|\gamma|=K}\left|c_{\beta \gamma}\right| \frac{(|\beta|+|\gamma|) !}{\{\widehat{\boldsymbol{s}} \cdot(\beta, \gamma)\} !}\right) T^{K} \\
& \quad+\left|f_{K+1}\right|\left(T, \ldots, T, C_{1} W,\left\{C_{2} \frac{d}{d T}\left(T \frac{d}{d T}\right)^{j-1} W\right\}_{(i, j)},\left\{C_{2} \frac{d}{d T} W\right\}_{k}\right)
\end{aligned}
$$

where $C_{1}=C C_{0}, C_{2}=M C_{0}$.

Let us explain how the equation (3.7) is derived from (3.6). By Lemmas 1 and 2 , we can show that a majorant relation $\mathcal{B}_{\widehat{\mathcal{S}}}(U)(y, z) \ll W(T)$ implies $\mathcal{B}_{\widehat{\boldsymbol{s}}}\left(P^{-1} U\right)(y, z) \ll C W(T)$ and

$$
\begin{aligned}
& \mathcal{B}_{\widehat{\boldsymbol{s}}}\left\{f_{K+1}\left(y, z, P^{-1} U, \partial_{y} P^{-1} U, \partial_{z} P^{-1} U\right)\right\} \\
& \quad \ll\left|f_{K+1}\right|\left(T, \ldots, T, C_{1} W,\left\{C_{2} \frac{d}{d T}\left(T \frac{d}{d T}\right)^{j-1} W\right\}_{(i, j)},\left\{C_{2} \frac{d}{d T} W\right\}_{k}\right) .
\end{aligned}
$$

Indeed, to prove this, it is sufficient to notice that $\mathcal{B}_{\widehat{\boldsymbol{s}}}\left(U^{2}\right) \ll\left\{C_{0} \mathcal{B}_{\widehat{\boldsymbol{s}}}(|U|)\right\}^{2}$ by $C_{0} \geq 1$, etc., and that

$$
\begin{aligned}
& \mathcal{B}_{\widehat{\boldsymbol{s}}}\left(y^{\beta} z^{\gamma}\left(P^{-1} U\right)^{r} \prod_{i, j, k}\left(\partial_{y_{i, j}} P^{-1} U\right)^{\mu_{i, j}}\left(\partial_{z_{k}} P^{-1} U\right)^{\nu_{k}}\right) \\
& \ll y^{\beta} z^{\gamma}\left\{C_{0} \mathcal{B}_{\widehat{\boldsymbol{s}}}\left(\left|P^{-1} U\right|\right)\right\}^{r} \prod_{i, j, k}\left\{C_{0} \mathcal{B}_{\widehat{\boldsymbol{s}}}\left(\left|\partial_{y_{i, j}} P^{-1} U\right|\right)\right\}^{\mu_{i, j}}\left\{C_{0} \mathcal{B}_{\widehat{\boldsymbol{s}}}\left(\left|\partial_{z_{k}} P^{-1} U\right|\right)\right\}^{\nu_{k}} \\
& \ll T^{|\beta|+|\gamma|}\left(C C_{0} W\right)^{r} \prod_{i, j, k}\left\{M C_{0} \frac{d}{d T}\left(T \frac{d}{d T}\right)^{j-1} W\right\}^{\mu_{i, j}}\left\{M C_{0} \frac{d}{d T} W\right\}^{\nu_{k}} .
\end{aligned}
$$

Therefore by the above construction of the equation (3.7), the formal solution $W(T)$ of $(3.7)$ is a majorant series of $\mathcal{B}_{\widehat{\boldsymbol{s}}}(U)(y, z)$, that is,

$$
W(|y|+|z|) \gg \mathcal{B}_{\widehat{\boldsymbol{s}}}(U)(y, z) .
$$

For the equation (3.7), we have $W(|y|+|z|) \in \mathcal{G}_{y, z}^{\mathbf{1}_{n}(\sigma)}$, because we can prove the following result:

Lemma 3. Let $p$ be nonnegative real number and $D_{T}=d / d T(T \in \mathbf{C})$. We define the formal differentiation $\left(T D_{T}\right)^{p}$ by

$$
\left(T D_{T}\right)^{p}\left(T^{L}\right):=L^{p} T^{L} .
$$


We consider the following nonlinear equation:

$$
\left\{\begin{array}{l}
U(T)=a T^{K}+f_{K+1}\left(T, U,\left(T D_{T}\right)^{p} U,\left\{D_{T}\left(T D_{T}\right)^{s_{k}} U\right\}_{k=1,2, \ldots, n}\right), \\
U(T)=O\left(T^{K}\right)
\end{array}\right.
$$

where $a \neq 0, p \geq 0, s_{k} \geq 0(k=1,2, \ldots, n), K \geq 2$ and

$$
f_{K+1}(T, U, V, \xi)=\sum_{V(i, j, m, \alpha) \geq K+1} f_{i j m \alpha} T^{i} U^{j} V^{m} \xi^{\alpha} .
$$

Here $V(i, j, m, \alpha)=i+K j+K m+(K-1)\left(\alpha_{1}+\cdots+\alpha_{n}\right)$ denotes the vanishing order of $T^{i} U^{j}\left\{\left(T D_{T}\right)^{p} U\right\}^{m} \prod_{k=1}^{n}\left\{D_{T}\left(T D_{T}\right)^{s_{k}} U\right\}^{\alpha_{k}}$. Then the equation (3.10) has a unique formal solution which belongs to $\mathcal{G}_{T}^{1+\sigma}$ with

$$
\sigma=\max _{i, j, m, \alpha}\left\{\frac{A(i, j, m, \alpha)}{V(i, j, m, \alpha)-K} ; f_{i j m \alpha} \neq 0\right\}
$$

where

$$
A(i, j, m, \alpha)=\left\{\begin{array}{cl}
\max \left\{p, \max \left\{s_{k}+1 ; \alpha_{k} \neq 0\right\}\right\} & \text { if }|\alpha| \geq 1, m \geq 1 \\
\max \left\{s_{k}+1 ; \alpha_{k} \neq 0\right\} & \text { if }|\alpha| \geq 1, m=0 \\
p & \text { if }|\alpha|=0, m \geq 1 \\
0 & \text { if }|\alpha|=m=0
\end{array}\right.
$$

We remark that $A(i, j, m, \alpha)$ denotes the maximal order of differentiation in the term $T^{i} U^{j}\left\{\left(T D_{T}\right)^{p} U\right\}^{m} \prod_{k=1}^{n}\left\{D_{T}\left(T D_{T}\right)^{s_{k}} U\right\}^{\alpha_{k}}$.

Now we return to the proof of Proposition 1. In our majorant equation (3.7), the maximal order of differentiation in each term is given by $A(\mu, \nu)$ which appeared in the statement of Proposition 1, and the difference of vanishing order of each term and that of $W(T)$ is given by

$$
|\beta|+|\gamma|+K r+(K-1)(|\mu|+|\nu|)-K
$$

Therefore, by Lemma 3 , we have $W(T) \in \mathcal{G}_{T}^{1+\sigma}$.

By Lemma 1 (ii), the following majorant relation holds:

$$
\mathcal{B}_{\widehat{\boldsymbol{s}}}(v)(y, z)=\mathcal{B}_{\widehat{\boldsymbol{s}}}\left(P^{-1} U\right)(y, z) \ll C W(|y|+|z|) \in \mathcal{G}_{y, z}^{\mathbf{1}_{n}(\sigma)}
$$

By Remark 1 (iii), we have $u(y, z) \in \mathcal{G}_{y, z}^{\widehat{\widehat{\boldsymbol{s}}}+\mathbf{1}_{n}(\sigma)-\mathbf{1}_{n}}=\mathcal{G}_{y, z}^{\boldsymbol{s}}$.

Thus Proposition 1 is proved. 


\section{§3.2. Proof of Proposition 2}

First we give the following lemma:

Lemma 4. (i) For all $L \geq 2$, the operator $P=\Lambda+\Delta: \mathbf{C}[y]_{L} \longrightarrow \mathbf{C}[y]_{L}$ is invertible.

(ii) Let $u_{L}(y), f_{L}(y) \in \mathbf{C}[y]_{L}$. We consider the following equation:

$$
P u_{L}(y)=f_{L}(y)
$$

Let a majorant relation $\mathcal{B}_{\widehat{\boldsymbol{t}}}\left(f_{L}\right)(y) \ll F_{L} \times T^{L}\left(F_{L} \geq 0\right)$ hold where $T=|y|=$ $\sum_{i=1}^{N} \sum_{j=1}^{k_{i}} y_{i, j}$ and $\widehat{\boldsymbol{t}}=\left(\boldsymbol{t}^{1}, \ldots, \boldsymbol{t}^{N}\right)$ which appeared in Proposition 2. Then there exists a positive constant $C>0$ independent of $L$ such that

$$
\begin{aligned}
\mathcal{B}_{\widehat{\boldsymbol{t}}}\left(u_{L}\right)(y) & =\mathcal{B}_{\hat{\boldsymbol{t}}}\left(P^{-1} f_{L}\right)(y) \\
& \ll C L^{-p} F_{L} \times T^{L}=: C\left(T \frac{d}{d T}\right)^{-p}\left(F_{L} \times T^{L}\right),
\end{aligned}
$$

where $p<1$ is the constant which appeared in (1.7).

We take $P v(y)=U(y)$ as a new unknown function. Then $U(y)$ satisfies the following equation:

$$
\left\{\begin{array}{l}
U(y)=\sum_{|\alpha|=K} c_{\alpha} y^{\alpha}+f_{K+1}\left(y, P^{-1} U(y), \partial_{y} P^{-1} U(y)\right), \\
U(y)=O\left(|y|^{K}\right) .
\end{array}\right.
$$

By the same argument as in the proof of Lemma 2 (ii), we have

$$
\mathcal{B}_{\widehat{\boldsymbol{t}}}\left(\partial_{y_{i, j}} P^{-1} U\right)(y) \ll M \frac{d}{d T}\left(T \frac{d}{d T}\right)^{s_{i, j}-p-1} W(T),
$$

where $s_{i, j}=j-p(j-1)$ for $i=1,2, \ldots, N, j=1,2, \ldots, k_{i}$.

Then the following equation gives a majorant equation for $\mathcal{B}_{\widehat{\boldsymbol{t}}}(U)(y)$ which is obtained from (3.12) by the $\widehat{\boldsymbol{t}}$-Borel transformation in the same way as in obtaining the equation (3.7).

$$
\begin{aligned}
& W(T)=\left(\sum_{|\alpha|=K}\left|c_{\alpha}\right| \frac{|\alpha| !}{\widehat{\boldsymbol{t}} \cdot \alpha) !}\right) T^{K} \\
& +\left|f_{K+1}\right|\left(T, \cdots, T, C_{1}\left(T \frac{d}{d T}\right)^{\max \{0,-p\}} W,\left\{C_{2} \frac{d}{d T}\left(T \frac{d}{d T}\right)^{s_{i, j}-p-1} W\right\}\right) .
\end{aligned}
$$


Here $C_{1}=C C_{0}$ and $C_{2}=M C_{0}$, where $C, C_{0}$ and $M$ are the positive constants which appeared in Lemma 4, Lemma 2 (i) and (3.13), respectively. This means that

$$
\mathcal{B}_{\widehat{\boldsymbol{t}}}(U)(y) \ll W(|y|)
$$

By Lemma 3, the formal solution $W(|y|)$ of (3.14) belongs to the Gevrey class $\mathcal{G}_{y}^{\mathbf{1}_{n}(\sigma)}$ where $\sigma$ is the constant which appeared in Proposition 2. Therefore by (3.11) we have

$$
\mathcal{B}_{\widehat{\boldsymbol{t}}}(v)(y)=\mathcal{B}_{\widehat{\boldsymbol{t}}}\left(P^{-1} U\right)(y) \ll C\left(T \frac{d}{d T}\right)^{-p} W(T) \in \mathcal{G}_{y}^{\mathbf{1}_{n}(\sigma)} .
$$

By Remark 1 (iii), the Gevrey order $s$ of $v(y)$ is estimated by

$$
\boldsymbol{s}=\widehat{\boldsymbol{t}}+\mathbf{1}_{n}(\sigma)-\mathbf{1}_{n}=\left(\boldsymbol{t}^{1}(\sigma), \ldots, \boldsymbol{t}^{N}(\sigma)\right) .
$$

Thus the proof is completed.

\section{§4. Proof of Lemmas}

\section{§4.1. Proof of Lemma 1}

By the lexicographic order of the basis of homogeneous polynomials of degree $L$ in $y=\left\{y_{i j}\right\}$ and $z=\left\{z_{k}\right\}$, the matrix representation of $P=$ $\sum_{i=1}^{p} \sum_{j=1}^{n_{i}-1} \delta y_{i, j+1} \partial_{y_{i, j}}+c$ is given by a triangle matrix as follows:

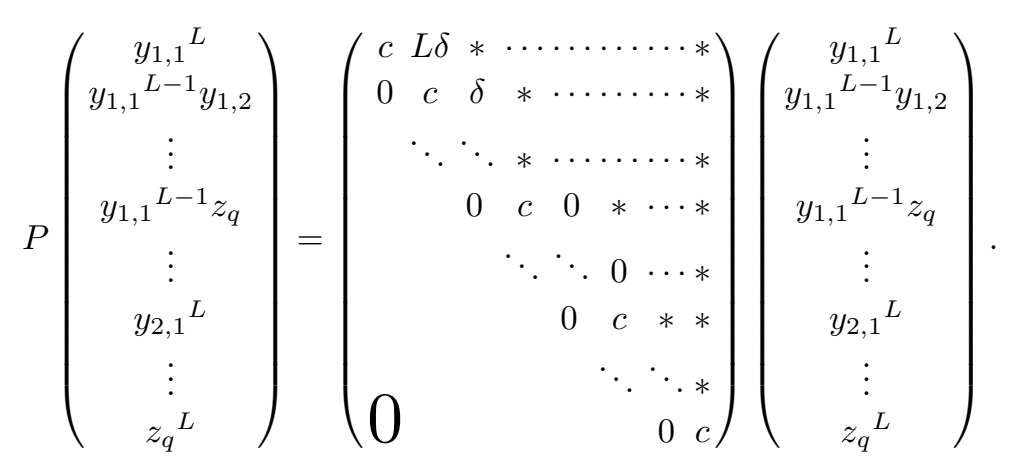

Therefore $P$ is an invertible operator for all $L \geq K$, since $c \neq 0$.

Thus the invertibility is proved.

For $f_{L}(y, z)=\sum_{|\beta|+|\gamma|=L} f_{\beta \gamma} y^{\beta} z^{\gamma} \in \mathbf{C}[y, z]_{L}$ and $s \in\left(\mathbf{R}_{\geq 1}\right)^{n}$, we introduce the $s$ norm of $f_{L}$ by

$$
\left\|f_{L}\right\|_{s}=\inf \left\{M>0 ; \mathcal{B}_{\boldsymbol{s}}\left(f_{L}\right)(y, z) \ll M \times(|y|+|z|)^{L}\right\} .
$$


Remark 3. (i) If a majorant relation $\mathcal{B}_{\boldsymbol{s}}\left(f_{L}\right)(y, z) \ll F_{L} \times(|y|+|z|)^{L}$ holds, then we have $\left\|f_{L}\right\|_{s} \leq F_{L}$.

(ii) The definition of the $s$ norm of $f_{L}$ is equivalent to the following:

$$
\left\|f_{L}\right\|_{s}=\max _{|\beta|+|\gamma|=L}\left\{\left|f_{\beta \gamma}\right| \frac{\beta ! \gamma !}{\{s \cdot(\beta, \gamma)\} !}\right\}
$$

where $\beta !=\prod_{i=1}^{p} \prod_{j=1}^{n_{i}} \beta_{i, j}$ ! and $\gamma !=\gamma_{1} ! \cdots \gamma_{q}$ !.

Let $e(i, j)$ be a vector defined by $e(i, j)=\left(\mathbf{e}^{1}, \ldots, \mathbf{e}^{p}\right) \in \mathbf{N}^{n_{1}+\cdots+n_{p}}$ where $\mathbf{e}^{k}=(0,0, \ldots, 0) \in \mathbf{N}^{n_{k}}$ for $k \neq i$ and $e_{i j}=1, e_{i l}=0(l \neq j)$ for $\mathbf{e}^{i}=$ $\left(e_{i l}\right)_{l=1,2, \ldots, n_{i}}$. Since

$$
y_{i, j+1} \partial_{y_{i, j}} f_{L}(y, z)=\sum_{|\beta|+|\gamma|=L} \beta_{i, j} f_{\beta \gamma} y^{\beta-e(i, j)+e(i, j+1)} z^{\gamma},
$$

we see that the $\widehat{\boldsymbol{s}}$ norm of $y_{i, j+1} \partial_{y_{i, j}} f_{L}(y, z)$ is estimated by

$$
\begin{aligned}
\left\|y_{i, j+1} \partial_{y_{i, j}} f_{L}\right\|_{\widehat{\boldsymbol{s}}} & =\max _{|\beta|+|\gamma|=L}\left\{\left|f_{\beta \gamma}\right| \frac{\beta_{i, j+1} \beta ! \gamma !}{\{\widehat{\boldsymbol{s}} \cdot(\beta-e(i, j)+e(i, j+1), \gamma)\} !}\right\} \\
& =\max _{|\beta|+|\gamma|=L}\left\{\left|f_{\beta \gamma}\right| \frac{\beta_{i, j+1} \beta ! \gamma !}{\{\widehat{\boldsymbol{s}} \cdot(\beta, \gamma)+1\} !}\right\} \\
& \leq \max _{|\beta|+|\gamma|=L}\left\{\left|f_{\beta \gamma}\right| \frac{\beta ! \gamma !}{\{\widehat{\boldsymbol{s}} \cdot(\beta, \gamma)\} !}\right\}=\left\|f_{L}\right\|_{\widehat{\boldsymbol{s}}},
\end{aligned}
$$

that is, the operator norm of $y_{i, j+1} \partial_{y_{i, j}}$ is estimated by $\left\|y_{i, j+1} \partial_{y_{i, j}}\right\|_{\widehat{\boldsymbol{s}}} \leq 1$. This implies

$$
\left\|\sum_{i=1}^{p} \sum_{j=1}^{n_{i}-1} \delta y_{i, j+1} \partial_{y_{i, j}}\right\|_{\widehat{s}} \leq\left(n_{1}+\cdots+n_{p}\right)|\delta| .
$$

As mentioned at Remark 2 in Section 2, we may assume $|\delta|$ to be as small as we want. Therefore we assume that $|\delta| \leq|c| /\left\{2\left(n_{1}+\cdots+n_{p}\right)\right\}$. By this inequality and Neumann's series expansion of $P^{-1}$, we have

$$
\left\|P^{-1}\right\|_{\widehat{s}} \leq \frac{|c|^{-1}}{1-\left\|\sum_{i=1}^{p} \sum_{j=1}^{n_{i}-1} c^{-1} \delta y_{i, j+1} \partial_{y_{i, j}}\right\|_{\widehat{\boldsymbol{s}}}} \leq \frac{2}{|c|} .
$$

Moreover, by Remark 3, we have

$$
\begin{aligned}
\mathcal{B}_{\widehat{\boldsymbol{s}}}\left(u_{L}\right)(y, z) & =\mathcal{B}_{\widehat{\boldsymbol{s}}}\left(P^{-1} f_{L}\right)(y, z) \\
& \ll\left\|\left.P^{-1}\right|_{\widehat{\boldsymbol{s}}} \times\right\| f_{L} \|_{\widehat{\boldsymbol{s}}} \times(|y|+|z|)^{L} \ll \frac{2}{|c|} F_{L} \times(|y|+|z|)^{L} .
\end{aligned}
$$




\section{§4.2. Proof of Lemma 2}

First we shall prove Lemma 2 (i). we have

Let $u(y, z)=\sum_{|\beta|+|\gamma| \geq 0} u_{\beta \gamma} y^{\beta} z^{\gamma}$ and $v(y, z)=\sum_{|\beta|+|\gamma| \geq 0} v_{\beta \gamma} y^{\beta} z^{\gamma}$. Then

$$
\begin{aligned}
& \mathcal{B}_{\widehat{\boldsymbol{s}}}(u v)(y, z)=\sum_{|\beta|+|\gamma| \geq 0}\left(\sum_{\substack{\beta^{1}+\beta^{2}=\beta \\
\gamma^{1}+\gamma^{2}=\gamma}} u_{\beta^{1} \gamma^{1}} v_{\beta^{2} \gamma^{2}} \frac{(|\beta|+|\gamma|) !}{\{\widehat{\boldsymbol{s}} \cdot(\beta, \gamma)\} !}\right) y^{\beta} z^{\gamma}, \\
& \mathcal{B}_{\widehat{\boldsymbol{s}}}(|u|)(y, z) \mathcal{B}_{\widehat{\boldsymbol{s}}}(|v|)(y, z) \\
& =\sum_{|\beta|+|\gamma| \geq 0}\left(\sum_{\substack{\beta^{1}+\beta^{2}=\beta \\
\gamma^{1}+\gamma^{2}=\gamma}}\left|u_{\beta^{1} \gamma^{1}}\right|\left|v_{\beta^{2} \gamma^{2}}\right| \frac{\left(\left|\beta^{1}\right|+\left|\gamma^{1}\right|\right) !}{\left\{\widehat{\boldsymbol{s}} \cdot\left(\beta^{1}, \gamma^{1}\right)\right\} !} \frac{\left(\left|\beta^{2}\right|+\left|\gamma^{2}\right|\right) !}{\left\{\widehat{\boldsymbol{s}} \cdot\left(\beta^{2}, \gamma^{2}\right)\right\} !}\right) y^{\beta} z^{\gamma} .
\end{aligned}
$$

We shall prove that there exists a positive constant $C_{0} \geq 1$ independent of $\beta$, $\gamma, \beta^{j}$ and $\gamma^{j}(j=1,2)$ such that

$$
\frac{(|\beta|+|\gamma|) !}{\{\widehat{\boldsymbol{s}} \cdot(\beta, \gamma)\} !} \leq C_{0} \frac{\left(\left|\beta^{1}\right|+\left|\gamma^{1}\right|\right) !}{\left\{\widehat{\boldsymbol{s}} \cdot\left(\beta^{1}, \gamma^{1}\right)\right\} !} \frac{\left(\left|\beta^{2}\right|+\left|\gamma^{2}\right|\right) !}{\left\{\widehat{\boldsymbol{s}} \cdot\left(\beta^{2}, \gamma^{2}\right)\right\} !}
$$

for all $\beta, \gamma, \beta^{j}$ and $\gamma^{j}$ with $\beta^{1}+\beta^{2}=\beta$ and $\gamma^{1}+\gamma^{2}=\gamma$ satisfying $\left|\beta^{j}\right|+\left|\gamma^{j}\right| \geq 1$ for $j=1$, 2. In fact, this implies Lemma 2 (i), because if $\left|\beta^{1}\right|=\left|\gamma^{1}\right|=0$ or $\left|\beta^{2}\right|=\left|\gamma^{2}\right|=0$, it is sufficient to take $C_{0}=1$.

By the relation between the Gamma functions and the Beta functions, we have

$$
\frac{\left\{\widehat{\boldsymbol{s}} \cdot\left(\beta^{1}, \gamma^{1}\right)\right\} !\left\{\widehat{\boldsymbol{s}} \cdot\left(\beta^{2}, \gamma^{2}\right)\right\} !}{\{\widehat{\boldsymbol{s}} \cdot(\beta, \gamma)\} !}=\{\widehat{\boldsymbol{s}} \cdot(\beta, \gamma)+1\} \cdot B\left(\widehat{\boldsymbol{s}} \cdot\left(\beta^{1}, \gamma^{1}\right)+1, \widehat{\boldsymbol{s}} \cdot\left(\beta^{2}, \gamma^{2}\right)+1\right)
$$

where $B(p, q)$ denotes the Beta function defined by

$$
B(p+1, q+1)=\int_{0}^{1} t^{p}(1-t)^{q} d t
$$

We remark that if $1 \leq p \leq p^{\prime}$ and $1 \leq q \leq q^{\prime}$, then $B\left(p^{\prime}+1, q^{\prime}+1\right) \leq$ $B(p+1, q+1)$ holds. By this inequality, we have

$$
\begin{aligned}
& \frac{\left\{\widehat{\boldsymbol{s}} \cdot\left(\beta^{1}, \gamma^{1}\right)\right\} !\left\{\widehat{\boldsymbol{s}} \cdot\left(\beta^{2}, \gamma^{2}\right)\right\} !}{\{\widehat{\boldsymbol{s}} \cdot(\beta, \gamma)\} !} \\
& \leq|\widehat{\boldsymbol{s}}|(|\beta|+|\gamma|+1) \cdot B\left(\left|\beta^{1}\right|+\left|\gamma^{1}\right|+1,\left|\beta^{2}\right|+\left|\gamma^{2}\right|+1\right) \\
& \quad=|\widehat{\boldsymbol{s}}| \frac{\left(\left|\beta^{1}\right|+\left|\gamma^{1}\right|\right) !\left(\left|\beta^{2}\right|+\left|\gamma^{2}\right|\right) !}{(|\beta|+|\gamma|) !},
\end{aligned}
$$


where $|\widehat{\boldsymbol{s}}|$ denotes the maximum of components of $\widehat{\boldsymbol{s}}$. This implies Lemma 2 (i).

For the proof of Lemma 2 (ii), we prepare the following lemma:

Lemma 5. For an arbitrary $\widetilde{\boldsymbol{s}}=\left\{s_{l}\right\} \in\left(\mathbf{R}_{\geq 1}\right)^{n}$ and an arbitrary power series $f(t)=\sum_{|\alpha| \geq 0} f_{\alpha} t^{\alpha}\left(t=\left(t_{1}, \ldots, t_{n}\right)\right)$, there exists a positive constant $M_{0}>0$ independent of $l=1,2, \ldots, n$ such that the following majorant relation holds:

$$
\mathcal{B}_{\widetilde{\boldsymbol{s}}}\left(\partial_{t_{l}} f\right)(t) \ll M_{0} \partial_{t_{l}}\left(t \cdot \partial_{t}\right)^{s_{l}-1} \mathcal{B}_{\widetilde{\boldsymbol{s}}}(|f|)(t),
$$

where $t \cdot \partial_{t}=t_{1} \partial_{t_{1}}+\cdots+t_{n} \partial_{t_{n}}$ and for $s \geq 0$ the operator $\left(t \cdot \partial_{t}\right)^{s}$ is defined by

$$
\left(t \cdot \partial_{t}\right)^{s} t^{\alpha}:=|\alpha|^{s} t^{\alpha}
$$

Proof. The both sides of (4.4) are calculated by

$$
\begin{aligned}
\text { Left hand side of }(4.4) & =\sum_{|\alpha| \geq 1} f_{\alpha} \frac{\alpha_{l}(|\alpha|-1) !}{\{\widetilde{\boldsymbol{s}} \cdot(\alpha-e(l))\} !} t^{\alpha-e(l)} \\
& =\sum_{|\alpha| \geq 1} f_{\alpha} \frac{\alpha_{l}(|\alpha|-1) !}{\left(\widetilde{\boldsymbol{s}} \cdot \alpha-s_{l}\right) !} t^{\alpha-e(l)}, \\
\text { Right hand side of }(4.4) & =M_{0} \sum_{|\alpha| \geq 1}\left|f_{\alpha}\right| \frac{\alpha_{l}|\alpha|^{s_{l}-1}|\alpha| !}{(\widetilde{\boldsymbol{s}} \cdot \alpha) !} t^{\alpha-e(l)},
\end{aligned}
$$

where $e(l)$ is an $n$-dimensional vector whose $l$-component is equal to 1 and the other components are equal to 0 . Hence, the majorant relation (4.4) follows from the following inequality:

$$
\frac{1}{\left(\widetilde{\boldsymbol{s}} \cdot \alpha-s_{l}\right) !} \leq M_{0} \times \frac{|\alpha|^{s_{l}}}{(\widetilde{\boldsymbol{s}} \cdot \alpha) !},
$$

where $M_{0}$ is a positive constant. Let us show (4.5).

- The case of $\widetilde{\boldsymbol{s}} \cdot \alpha-s_{l}=0$, that is, $\alpha=e(l)$. It is sufficient to put $M_{0}=\max _{l=1,2, \ldots, n}\left\{s_{l} !\right\}$.

- The case of $\widetilde{\boldsymbol{s}} \cdot \alpha-s_{l} \geq 1$. We put $a=\widetilde{\boldsymbol{s}} \cdot \alpha$ for simplicity. By the Stirling formula, there exist positive constants $C_{1}$ and $C_{2}$ independent of $\alpha$ and $\widetilde{\boldsymbol{s}}$ such that

$$
a ! \leq C_{1} a^{a+1 / 2} e^{-a}, \quad\left(a-s_{l}\right) ! \geq C_{2}\left(a-s_{l}\right)^{a-s_{l}+1 / 2} e^{-a+s_{l}} .
$$

These imply

$$
\frac{a !}{\left(a-s_{l}\right) !} \leq \frac{C_{1} a^{a+1 / 2} e^{-a}}{C_{2}\left(a-s_{l}\right)^{a-s_{l}+1 / 2} e^{-a+s_{l}}}=\frac{C_{3}}{e^{s_{l}}}\left(\frac{a}{a-s_{l}}\right)^{a-s_{l}+1 / 2} \times a^{s_{l}}
$$


where $C_{3}=C_{1} / C_{2}$. By noticing an inequality

$$
\left(\frac{a}{a-s_{l}}\right)^{a-s_{l}+1 / 2}=\left(1+\frac{s_{l}}{a-s_{l}}\right)^{a-s_{l}} \sqrt{1+\frac{s_{l}}{a-s_{l}}} \leq e^{s_{l}} \sqrt{1+s_{l}},
$$

we obtain

$$
\frac{a !}{\left(a-s_{l}\right) !} \leq C_{4}\left(\max _{j=1,2, \ldots, n}\left\{s_{j}\right\}\right)^{s_{l}}|\alpha|^{s_{l}}
$$

where $C_{4}=\max \left\{C_{3} \sqrt{1+s_{j}} ; j=1,2, \ldots, n\right\}$.

It is enough to take $M_{0}=\max _{l=1,2, \ldots, n}\left\{s_{l} !, C_{4}\left(\max _{j=1,2, \ldots, n}\left\{s_{j}\right\}\right)^{s_{l}}\right\}$ in (4.5).

We return to the proof of Lemma 2 (ii). Let $\widetilde{\boldsymbol{s}}=\widehat{\boldsymbol{s}}=\left(\boldsymbol{s}^{1}, \ldots, \boldsymbol{s}^{p}, \mathbf{1}_{q}\right)$ and $T=t_{1}+\cdots+t_{n}$. By Lemmas 1 and 5 , the following majorant relation holds:

$$
\begin{aligned}
\mathcal{B}_{\widehat{\boldsymbol{s}}}\left(\partial_{t_{l}} P^{-1} u\right) & \ll M_{0} \partial_{t_{l}}\left(t \cdot \partial_{t}\right)^{s_{l}-1} \mathcal{B}_{\widehat{\boldsymbol{s}}}\left(\left|P^{-1} u\right|\right)(t) \\
& \ll C M_{0} \partial_{t_{l}}\left(t \cdot \partial_{t}\right)^{s_{l}-1} W(T) .
\end{aligned}
$$

Now it is sufficient to prove the following equality:

$$
\partial_{t_{l}}\left(t \cdot \partial_{t}\right)^{s_{l}-1} W(T)=\frac{d}{d T}\left(T \frac{d}{d T}\right)^{s_{l}-1} W(T) .
$$

Indeed, by putting $t=\left(\boldsymbol{y}^{1}, \ldots, \boldsymbol{y}^{p}, z\right)$ and $\widetilde{\boldsymbol{s}}=\widehat{\boldsymbol{s}}=\left(\boldsymbol{s}^{1}, \ldots, \boldsymbol{s}^{p}, \mathbf{1}_{q}\right)$, we obtain Lemma 2 (ii).

For $T^{L}=\left(t_{1}+\cdots+t_{n}\right)^{L}$, we have

$$
\partial_{t_{l}}\left(T^{L}\right)=\frac{d}{d T}\left(T^{L}\right), \quad\left(t \cdot \partial_{t}\right)^{s_{l}-1}\left(T^{L}\right)=\left(T \frac{d}{d T}\right)^{s_{l}-1}\left(T^{L}\right) .
$$

Thus we have

$$
\text { Left hand side of }(4.6)=\frac{d}{d T}\left(T \frac{d}{d T}\right)^{s_{l}-1} W(T)
$$

which shows (4.6).

\section{§4.3. Proof of Lemma 3}

Lemma 3 is proved in a similar manner to the proof of Theorem 1 in Shirai $[4]$.

Let $U(T)=\sum_{L \geq K} U_{L} T^{L}$ be a formal solution of (3.10). By substituting this into the equation (3.10), we have the following recursion formula:

$$
U_{K}=a,
$$


and for $L>K$

$$
U_{L}=\sum_{(*)} f_{i j m \alpha} \prod_{k=1}^{j} U_{M_{k}} \prod_{k=1}^{m}\left(L_{k}\right)^{p} U_{L_{k}} \prod_{k=1}^{n} \prod_{l=1}^{\alpha_{k}}\left(N_{k l}\right)^{s_{k}+1} U_{N_{k l}}
$$

where $\sum_{(*)}$ is taken over

$$
i+\sum_{k=1}^{j} M_{k}+\sum_{k=1}^{m} L_{k}+\sum_{k=1}^{n} \sum_{l=1}^{\alpha_{k}}\left(N_{k l}-1\right)=L \quad \text { and } \quad V(i, j, m, \alpha) \geq K+1 .
$$

Let $V_{L}=U_{L} /(L !)^{\sigma}$. Then $\left\{V_{L}\right\}$ satisfy

$$
V_{L}=\sum_{(*)} f_{i j m \alpha} G(i, j, m, \alpha) \prod_{k=1}^{j} V_{M_{k}} \prod_{k=1}^{m} V_{L_{k}} \prod_{k=1}^{n} \prod_{l=1}^{\alpha_{k}} V_{N_{k l}}
$$

where

$$
\begin{aligned}
G(i, j, m, \alpha)= & \prod_{k=1}^{m}\left(L_{k}\right)^{p} \prod_{k=1}^{n} \prod_{l=1}^{\alpha_{k}}\left(N_{k l}\right)^{s_{k}+1} \\
& \times \frac{\left(\prod_{k=1}^{j} M_{k} ! \prod_{k=1}^{m} L_{k} ! \prod_{k=1}^{n} \prod_{l=1}^{\alpha_{k}} N_{k l} !\right)^{\sigma}}{L !^{\sigma}} .
\end{aligned}
$$

We notice the following inequality which can be seen in Shirai [4, Lemma 6].

Lemma 6. If $m_{1}, \ldots, m_{d} \in \mathbf{N}$ satisfy $m_{j} \geq M(M \in \mathbf{N})$ for all $j=$ $1,2, \ldots, d$, then we have

$$
m_{1} ! \cdots m_{d} ! \leq(M !)^{d-1}\left(m_{1}+\cdots+m_{d}-(d-1) M\right) ! .
$$

By Lemma 6 , for an arbitrary $M \in \mathbf{N}, G(i, j, m, \alpha)$ is estimated by (4.9) $G(i, j, m, \alpha)$

$$
\begin{aligned}
\leq & \prod_{k=1}^{m}\left(L_{k}\right)^{p-M \sigma} \prod_{k=1}^{n} \prod_{l=1}^{\alpha_{k}}\left(N_{k l}\right)^{s_{k}+1-M \sigma} \\
& \times \frac{\left(\prod_{k=1}^{j}\left(M_{k}+M\right) ! \prod_{k=1}^{m}\left(L_{k}+M\right) ! \prod_{k=1}^{n} \prod_{l=1}^{\alpha_{k}}\left(N_{k l}+M\right) !\right)^{\sigma}}{L ! \sigma} \\
\leq & (K+M) !^{(j+m+|\alpha|-1) \sigma} \prod_{k=1}^{m}\left(L_{k}\right)^{p-M \sigma} \prod_{k=1}^{n} \prod_{l=1}^{\alpha_{k}}\left(N_{k l}\right)^{s_{k}+1-M \sigma} B^{\sigma},
\end{aligned}
$$


where

$$
B=\frac{\left(\sum_{k=1}^{j} M_{k}+\sum_{k=1}^{m} L_{k}+\sum_{k=1}^{n} \sum_{l=1}^{\alpha_{k}} N_{k l}+M-(j+m+|\alpha|-1) K\right) !}{\left(i+\sum_{k=1}^{j} M_{k}+\sum_{k=1}^{m} L_{k}+\sum_{k=1}^{n} \sum_{l=1}^{\alpha_{k}}\left(N_{k l}-1\right)\right) !}
$$

Let

$$
\begin{aligned}
& \Omega_{1}=\left\{(i, j, m, \alpha) ; \frac{A(i, j, m, \alpha)}{V(i, j, m, \alpha)-K}=\sigma\right\}, \\
& \Omega_{2}=\left\{(i, j, m, \alpha) ; \frac{A(i, j, m, \alpha)}{V(i, j, m, \alpha)-K}<\sigma\right\} .
\end{aligned}
$$

We remark that $\Omega_{1}$ is a finite set.

We put $M$ which is used in (4.9) by

$$
M=M(i, j, m, \alpha)= \begin{cases}V(i, j, m, \alpha)-K, & (i, j, m, \alpha) \in \Omega_{1}, \\ {\left[\frac{A(i, j, m, \alpha)}{\sigma}\right]+1,} & (i, j, m, \alpha) \in \Omega_{2},\end{cases}
$$

where $[\cdots]$ denotes the integral part of $\{\cdots\}$. By the definition of $\sigma$ and (4.11), we have

$$
\frac{A(i, j, m, \alpha)}{\sigma}<M \leq \frac{A(i, j, m, \alpha)}{\sigma}+1<V(i, j, m, \alpha)-K+1
$$

for $(i, j, m, \alpha) \in \Omega_{2}$. By this inequality, we have $M \leq V(i, j, m, \alpha)-K$ for all $(i, j, m, \alpha)$, because $M$ and $V(i, j, m, \alpha)-K+1$ are natural numbers. By the choice of $M$ as (4.11), we have

$$
\prod_{k=1}^{m}\left(L_{k}\right)^{p-M \sigma} \prod_{k=1}^{n} \prod_{l=1}^{\alpha_{k}}\left(N_{k l}\right)^{s_{k}+1-M \sigma} \leq 1, \quad \text { and } \quad B^{\sigma} \leq 1,
$$

for all $(i, j, m, \alpha)$, where $B$ is defined above as (4.10). This implies that

$$
\begin{aligned}
G(i, j, m, \alpha) & \leq(K+\max \{M(i, j, m, \alpha)\}) !^{(j+m+|\alpha|-1) \sigma} \\
& <\left\{(K+\max \{M(i, j, m, \alpha)\}) !^{\sigma}\right\}^{j+m+|\alpha|}=: C^{j+m+|\alpha|}
\end{aligned}
$$

holds for all $(i, j, m, \alpha)$. Therefore $V_{L}$ is estimated by

$$
\left|V_{L}\right| \leq \sum_{(*)}\left|f_{i j m \alpha}\right| \prod_{k=1}^{j} C\left|V_{M_{k}}\right| \prod_{k=1}^{m} C\left|V_{L_{k}}\right| \prod_{k=1}^{n} \prod_{l=1}^{\alpha_{k}} C\left|V_{N_{k l}}\right| .
$$


Finally, we consider the following functional equation:

$$
W(T)=\frac{|a|}{K ! \sigma} T^{K}+\left|f_{K+1}\right|(T, C W, C W,\{C W / T\}), \quad W(T)=O\left(T^{K}\right) .
$$

Then the unique existence of holomorphic solution $W(T)$ in a neighborhood of the origin follows by the implicit function theorem since the nonlinear part $\left|f_{K+1}\right|$ satisfies $\left|f_{K+1}\right|(T, C W, C W,\{C W / T\})=O\left(T^{K+1}\right)$. Moreover, by (4.13), we can see that

$$
W(T) \gg \sum_{L \geq K} V_{L} T^{L}=\sum_{L \geq K} \frac{U_{L}}{L ! \sigma} T^{L} .
$$

Thus Lemma 3 is proved.

\section{§4.4. Proof of Lemma 4}

The invertibility is easily proved by the same argument as in the proof of Lemma 1, so we omit the proof.

By the assumption (1.7), we have $\sum_{i=1}^{N} \sum_{j=1}^{k_{i}} \lambda_{i} \alpha_{i, j}+c \neq 0\left(c=f_{u}(0,0\right.$, $\left.\xi^{0}\right)$ ). Hence we easily see that $\Lambda$ is invertible on $\mathbf{C}[y]_{L}$ and the inverse is given by

$$
\Lambda^{-1} f_{L}(y)=\sum_{|\alpha|=L}\left(\sum_{i=1}^{N} \sum_{j=1}^{k_{i}} \lambda_{i} \alpha_{i, j}+c\right)^{-1} f_{\alpha} y^{\alpha} .
$$

By the definition of the $\widehat{\boldsymbol{t}}$ norm and (1.7), we have

$$
\left\|\Lambda^{-1} f_{L}\right\|_{\widehat{\boldsymbol{t}}} \leq \max _{|\alpha|=L}\left\{\frac{\left|f_{\alpha}\right|}{C L^{p}} \frac{\alpha !}{(\widehat{\boldsymbol{t}} \cdot \alpha) !}\right\}=\frac{1}{C L^{p}}\left\|f_{L}\right\|_{\widehat{\boldsymbol{t}}} .
$$

This implies

$$
\left\|\Lambda^{-1}\right\|_{\widehat{t}} \leq \frac{1}{C L^{p}}
$$

Moreover, by the same calculation as in Section 4.1, we have

$$
\begin{aligned}
\left\|y_{i, j+1} \partial_{y_{i, j}} f_{L}\right\|_{\widehat{\boldsymbol{t}}} & =\max _{|\alpha|=L}\left\{\left|f_{\alpha}\right| \frac{\alpha_{i, j}\{\alpha-e(i, j)+e(i, j+1)\} !}{\{\widehat{\boldsymbol{t}} \cdot(\alpha-e(i, j)+e(i, j+1))\} !}\right\} \\
& =\max _{|\alpha|=L}\left\{\left|f_{\alpha}\right| \frac{\alpha_{i, j+1} \alpha !}{\widehat{(\boldsymbol{t} \cdot \alpha+1-p) !}\}}\right. \\
& \leq \max _{|\alpha|=L}\left\{\left|f_{\alpha}\right| \frac{\alpha !}{\widehat{(\boldsymbol{t} \cdot \alpha-p) !}}\right\}
\end{aligned}
$$


By the same argument as in the proof of the inequality (4.5), we have

$$
\frac{1}{(\widehat{\boldsymbol{t}} \cdot \alpha-p) !} \leq \frac{C_{0} L^{p}}{(\widehat{\boldsymbol{t}} \cdot \alpha) !}
$$

with some $C_{0}>0$ independent of $L$. This implies that the operator norm of $y_{i, j+1} \partial_{y_{i, j}}$ is estimated as $\left\|y_{i, j+1} \partial_{y_{i, j}}\right\|_{\widehat{\boldsymbol{t}}} \leq C_{0} L^{p}$, that is,

$$
\|\Delta\|_{\widehat{\boldsymbol{t}}} \leq \sum_{i=1}^{N} \sum_{j=1}^{k_{i}-1}|\delta| C_{0} L^{p} \leq|\delta| n C_{0} L^{p} .
$$

Since we can take $|\delta|$ as small as we want, we may assume that $\left\|\Lambda^{-1} \Delta\right\|_{\widehat{t}} \leq 1 / 2$. Then we have

$$
\left\|P^{-1}\right\|_{\widehat{\boldsymbol{t}}} \leq \frac{\left\|\Lambda^{-1}\right\|_{\widehat{\boldsymbol{t}}}}{1-\left\|\Lambda^{-1} \Delta\right\|_{\widehat{\boldsymbol{t}}}} \leq \frac{2}{C L^{p}}
$$

This inequality implies

$$
\mathcal{B}_{\widehat{\boldsymbol{t}}}\left(u_{L}\right)(y)=\mathcal{B}_{\widehat{\boldsymbol{t}}}\left(P^{-1} f_{L}\right)(y) \ll \frac{2}{C}\left(T \frac{d}{d T}\right)^{-p} F_{L} \times T^{L} .
$$

\section{References}

[1] Gérard, R. and Tahara, H., Singular nonlinear partial differential equations, Vieweg, 1996.

[2] Hibino, M., Divergence property of formal solutions for singular first order linear partial differential equations, Publ. RIMS, Kyoto Univ., 35 (1999), 893-919.

[3] Miyake, M. and Shirai, A., Convergence of formal solutions of first order singular nonlinear partial differential equations in complex domain, Ann. Polon. Math., 74 (2000), 215-228.

[4] Shirai, A., Maillet type theorem for nonlinear partial differential equations and Newton polygons, J. Math. Soc. Japan., 53 (2001), 565-587.

[5] Yamazawa, H., Newton polyhedrons and a formal Gevrey space of double indices for linear partial differential operators, Funkcial. Ekvac., 41 (1998), 337-345.

[6] Yamazawa, H., Formal Gevrey class of formal power series solution for singular first order linear partial differential operators, Tokyo J. Math., 23 (2000), 537-561. 